\title{
A System Architecture for CAD-Based Robotic Assembly with Sensor-Based Skills
}

\author{
Yudha Pane, Student Member, IEEE, Mathias Hauan Arbo, Member, IEEE, Erwin Aertbeliën, and \\ Wilm Decré, Member, IEEE
}

\begin{abstract}
Specifying assembly tasks in CAD-level is a promising approach to intuitively program complex robot skills. In this paper, a three-layered system architecture is presented to generate sensor-based robot skills from an assembly task instance. The architecture consists of an application layer where the user instantiates assembly tasks by specifying CAD constraints between geometric primitives pairs. A process layer infers the most suitable robot skills and their appropriate parameters. This inference is made possible by reasoning on a knowledge database represented as an ontology. The ontology contains semantic models of relevant classes such as tasks, skills, and geometric primitives as well as the relations between them. A control layer executes the sensor-based skills in real time using the eTaSL programming framework. A software implementation for the three layers is presented. The application layer is implemented in FreeCAD while the process layer consists of an OWL ontology, a Prolog-based reasoner and fuzzy inference to correctly select the skill and generate its parameters. In the control layer, the instantiated eTaSL skills execute the assembly tasks by sending an optimized control command to the robot. The system is validated on two challenging assembly cases with two distinct robot types, thus demonstrating the system's capability across different scenarios.
\end{abstract}

Note to Practitioners-The widespread use of CAD models for describing parts assembly has motivated the research community to create systems that automatically generate robot programs satisfying the assembly goal. While most of the existing literature focuses on generating the assembly sequence, this paper deals with the aspect of translation from CAD-level assembly specification to executable robot motion, also called skills. The paper systematically addresses the problem by dividing it into different layers and solving them separately. Parameters that influence the successful execution of an assembly task are identified and categorized into application-related and processrelated parameters. Different inference techniques are employed to address each parameter category. Experimental results show that the proposed system is able to successfully generate and execute robot skills for assembly scenarios of an air compressor and an electric motor.

Index Terms-Assembly systems, robot programming, computer aided software engineering, force control, knowledge based systems.

\section{INTRODUCTION}

Y. Pane, E. Aertbeliën, and W. Decré are with the Department of Mechanical Engineering, Katholieke Universiteit Leuven and Core Lab ROB, Flanders Make@KU Leuven..

M. H. Arbo is with Department of Engineering Cybernetics, Norwegian University of Science and Technology.

The work reported in this paper was supported by Flanders Make ICON FINROP (Fast and Intuitive Robot Programming) and SBO PROUD (Programming by User Demonstration) in Belgium and the centre for research based innovation SFI Manufacturing in Norway. The work is partially funded by the Research Council of Norway under contract number 237900.

Manuscript received May 2019.
$\mathbf{R}$ ECENT advancement in robotics technology provides the opportunity to perform complex tasks that were previously considered unsuitable for automation. Examples are tasks that require on-line sensor interaction as well as humanrobot cooperation. The increasingly more complex tasks, however, also mean an increased programming effort. One of the main reasons is that task specification and parameterization require sufficient expertise and is often written in robotspecific languages. This traditional way of programming is excellent for specifying simple tasks such as point-to-point motion, but unsuitable for complex tasks such as force-based assembly. Not only does this make it difficult for non-robot experts to program challenging tasks, it also increases the associated cost. Therefore, there is a need for more intuitive methods to program robot tasks.

Computer-Aided Design (CAD) is a rich source of information that is widely used by manufacturing companies and can potentially help reduce the robot programming effort. The information includes part dimensions, geometric features, mating descriptions, etc. In this work, we are interested in exploiting CAD information to solve a particular class of robotic tasks, namely assembly. Although this problem has been addressed in the research literature, there are still challenges to be solved. Our system aims to improve upon existing works by introducing a more systematic grounding from assembly specification in CAD-level to robot motion control. Inspired by the work of Perzylo et al. [1], we implemented an extended ontology which, additionally, covers force-based task classes. The ontology stores knowledge about different CAD primitives, assembly constraints, robot motion constraints, as well as their relations. By reasoning with this knowledge, suitable skills to execute a given assembly task can be deduced and suggested to the user.

In designing the system, we advocate for: a workpiece perspective, composability, and sensor integration. A workpiece perspective allows us to specify the task's constraints relative to the parts. This makes the system more intuitive for users with less robot expertise, and it gives a robot-agnostic formulation of what is to be achieved. Composability allows us to combine different models of the environment, workpiece, and robot. This property is important in order to improve reusability of the assembly program. Sensor integration, in particular for force control, is a key to support a larger class of assembly tasks.

The key contribution of this paper is the design of a system architecture and software implementation that facilitates instantiation of constraint-based skills to execute position and 
force-based assembly tasks specified with CAD semantics. For the skill implementation, we opted for the eTaSL framework [2] due to it being one of the state-of-the-art frameworks for constraint-based robot programming and its relatively mature software development. The grounding from the CAD-level assembly specification to executable skills is broken down into two main problems: skill selection and skill parameterization. The former deals with finding the suitable skills from the provided library for a given assembly task. To this end, we extend our initial work from [3] by adding a reasoning module implemented in Prolog to search through the ontology for the appropriate skills. Once a skill is chosen, it needs contextdependent parameters in order to be executable. These skillspecific parameters are split into two groups. One group, namely application parameters, are geometry-related and can be inferred from the CAD information. Examples of this class of parameters are assembly direction and insertion depth. The second group, termed process parameters, are difficult to completely determine in a model-based way, hence they are typically tuned. Parameters such as insertion force and antijamming dither belong to this parameter class. In this work, we propose a data-driven approach to estimate the appropriate process parameters using a fuzzy inference method. The resulting parameters are then used to instantiate an eTaSLbased skill. Using this architecture, complex force-controlled assembly tasks can be executed even though the skills rely on empirical parameters.

In our previous work [3], the mapping from CAD-level specification to concrete skills was done statically without involving any reasoning capability. This limits the reusability of the skills and puts more programming load on the user. The differentiating contributions of this article compared to our previous work are as follows. First, we introduce a knowledge database which is represented as an ontology to encode semantic information of CAD-compliant geometric models as well as force-based skill constraints. Secondly, we implement an inference module using logic programming to reason on top of the ontology in order to assist in grounding from CADlevel specification to suitable skills. Finally, we present more extensive use cases by showing a different assembly problem performed by a different robot setup.

This article is organized as follows. Section II summarizes the previous works related to our research. Section III describes the overview of the system architecture and defines tasks and skills. The theory behind the ontology knowledge database and the inference module is covered in Section IV. Section V explains the implementation detail of each system's component. This includes the software implementation of the CAD workbench and the inference module. The experimental validation on two different assembly scenarios are presented in Section VI. Section VII discusses the experimental results.

\section{RELATED WORKS}

One of the earliest works in exploiting CAD information to generate robotics assembly program was presented by Ambler and Popplestone [4], where they proposed a constraint-based description of pose relations between geometric features of assembly parts. Reference frames attached to the geometric features were used to define the end-product of assembly in terms of "fits" or "against" relationships. The relative pose between these frames was described by equality or inequality constraints.

AUTOPASS [5] is an early CAD-based assembly programming system that starts from a workpiece rather than a robotcentric perspective. AUTOPASS includes modules for precalculating trajectories that are executed by the robot with basic use of sensor data for ensuring the success of the assembly task. Archimedes 2 [6] describes an architecture where the CAD model and assembly description, sequence planning, and motion control execution are separated into modules. Archimedes 2 defines two forms of assembly tasks: mating and joining. Mating is establishing a relative positioning of the parts along a translation, rotation, or screw trajectory. Joining is attaching the part in its relative position by either gluing or welding, while using a predefined tool for the particular task. One of the most advanced systems is HighLAP [7] [8] where the CAD model is annotated with a small set of simple semantic assembly descriptions. The descriptions give constraints similar to Ambler and Popplestone that are used to find remaining degrees-of-freedom. HighLAP uses the task frame formalism to execute force-controlled motion [9]. Tasks are executed by a "net" of skill primitives, i.e. a finitestate machine of skills with sensor-based transition conditions. From the geometric constraint relations between the parts, tasks are classified e.g. as object placing or peg in hole, and the particular suitable net of skill primitives is employed [10].

The work by Perzylo et al. uses object-centric programming based on geometric constraints between parts to simplify the robot motion programming [11]. The comparison of the object-centric programming and classical teach-pendant based programming shows that the former is faster. The underlying framework is presented in [12] where Somani et al. proposes a robot programming framework specified with CAD semantics. The framework translates the inherently underspecified CAD constraints into a position-resolved optimization problem. The framework makes use of the Boundary Representation (BREP)-based ontology explained in [1] for annotation of geometric constraint relationships between CAD parts. By defining an ontology for geometric constraints and CAD primitives, other secondary information such as the motion null-spaces formed by constraints can be exploited to find the assembly paths. The work of Perzylo et al. is a part of the EU funded SMErobotics consortium [13] which aims to simplify production systems for small and medium-sized enterprises. The consortium includes a product-process-resource technology model [14] that allows process-level information such as welding voltage to be incorporated through the technology model. The research presented by the consortium approaches a wide range of manufacturing domains from assembly to welding, and includes a product-centric robot instructions and uncertainty-aware robot skills [15].

The aforementioned literature deals with a common problem: to ground a higher-level abstraction of task specification in the CAD-level into the lower-level abstraction of robot motion. Although they bring significant progress in solving 
the problem, there are still some limitations to be resolved. In the AUTOPASS language, assembly specification is refined into robot motion by using a dedicated compiler that composes functional modules. However, the language lacks semantics of CAD primitives and CAD-specific constraints as it was developed before the BREP-format became popular. This makes grounding to the corresponding robot motion more difficult due to the specification's ambiguity and reliance on outdated formats. Archimedes 2 presents results for positionbased assembly tasks, but does not treat force-based assembly problems which are often found in real-life assemblies. Furthermore, the system generates robot-specific function calls which implies robot vendor dependency, thus reducing its reusability. The framework proposed by Perzylo and Somani et al. covers more comprehensive classes of CAD primitives, assembly constraints, and their mapping to the corresponding robot motion constraints. However, the framework is limited to position-based assembly problems. The uncertainty-aware robot skills of Thomas et al., are generalizable skills that include force-controlled motions but are yet to be unified with the CAD assembly constraints of Perzylo et al. The HighLAP system aims to fully automate an assembly task by using contact formation graphs to eventually generate manipulation primitive nets. The disadvantage of such an approach is that it requires state estimation which is prone to errors due to measurement uncertainties and disturbances. Furthermore, constraints between two parts is specified with relations such as fits, against, and aligned. The semantics of these relations are typically not supported in modern CAD software.

Motions for assembly are easily defined w.r.t. the workpiece, environment, or robot using constraints, and the constraints are naturally composable. Mason's task frame formalism [16] presents an early work for describing fine motion in the task space by introducing the notion of natural and artificial constraints. It shows how different control modes can be applied independently along instantaneous task frame's directions, both in translation and rotation. In [17], De Schutter et al. describe a procedure to design a robot controller that can deal with sensor interactions and motion in contact, using a set of auxiliary frames to systematically describe the constraints. This approach is implemented in the software framework iTaSC [18]. Subsequently, expression graphs are used to simplify constraint-based programming leading to the specification language eTaSL [2]. eTaSL facilitates the specification of controller behavior using constraints that relate to geometry or sensor-input. Furthermore, it also allows specifying monitors that trigger events upon certain conditions such as success or failure. These events can be captured by discrete-time coordinators such as finite state machine (FSM) or behavior tree to facilitate transition between skills.

\section{OVERVIEW}

The system architecture is designed by following separation of concern principle, such that different level of abstraction is treated separately. We describe the three layers that constitute the proposed architecture as well as distinguish the notion of tasks and skills.

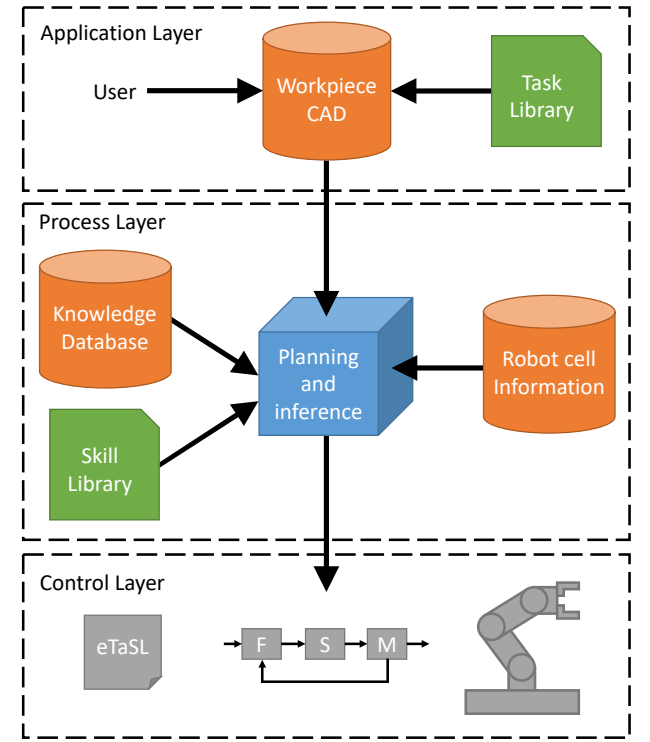

Fig. 1: The overall system architecture of the proposed system

\section{A. System Architecture}

The system architecture is outlined in Fig. 1 and is split up into three layers: the application layer, the process layer, and the control layer. In the application layer we have a workpiececentric view, where the user instantiates a task by selecting the desired template from the task library and annotating the CAD model in accordance to the task specification. The task library consists of predefined assembly tasks, each with its own task specification. After a task instance is created, it is passed on to the process layer. In the process layer, assembly is considered from the point of view of the workcell. The planning and inference module ensures appropriate assembly sequence selection and composition of the correct skills, as well as generating both the skills' application and process parameters. The application parameters can be extracted from the task instance's CAD model, e.g. the assembly direction or the insertion depth. Process parameters may relate to the CAD data but without a clear underlying model. They may be empirical or have a range of values that produce acceptable results e.g. insertion force or amplitude of the anti-jamming dither. Furthermore, robot cell information such as safety boundary and workspace limit can be taken into account so as to ensure task execution while respecting the workcell condition. For example, collision with the assembly workbench can be avoided by composing an inequality constraint to the end-effector pose such that it always operates above the workbench's surface. Finally, the skill instances that grounded the assembly tasks are executed in the control layer. Transition between the skills are handled with a finite state machine.

\section{B. Skill and Task}

Skill and task are sometimes used interchangeably in the robotics literature. In this paper, the terms refer to two different concepts. This follows the nomenclature presented in the previous paper [3] with a slight alteration from the increased CAD integration presented in this paper. 
A task is defined as a piece of work to be undertaken, while a skill is the robot's particular ability. An assembly task is described with a task specification which is composed of CAD constraints between two different parts. These constraints describe the desired end condition of the assembly. They conform to the mating constraints typically found in most well known CAD software. Examples of the supported assembly constraints are: concentric, coincident and parallel. An example of a task specification for a cylindrical object insertion is provided in Listing 1 . It specifies that the two cylindrical surfaces of the object and the hole should be concentric and the bottom planes of the two should be coincident.

Listing 1: Cylindrical object insert specification

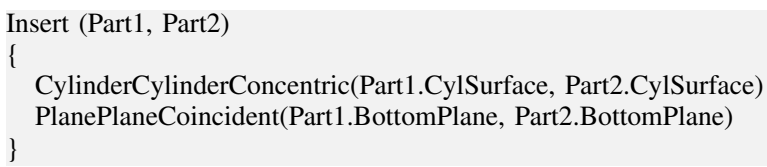

A skill is defined as an eTaSL specification that has: configuration, inputs, outputs, and events description. The configuration is a set of application and process parameters that are constant during the skill execution. The inputs bring information from continuous sources such as sensors. The outputs are mainly used for logging or analysis purposes. Monitors can trigger events that denote success or failure of the skill.

Since eTaSL is a constraint-based programming paradigm, a skill is composed of eTaSL constraints that are imposed on robot expressions such as joint positions or the end-effector pose. The eTaSL constraints that are used in our skills can be categorized as either kinematic-based or wrench-based. A kinematic-based constraint is used to control a given expression in position or velocity-level. A wrench-based constraint imposes certain force and torque values.

As Fig. 1 shows, in our proposed system, the possible tasks and skills are predefined in the task and skill library, respectively. The task library can be extended by the user by simply defining a new composition of CAD constraints. Meanwhile, extending the skill library is delegated to the skill developers who are typically robot experts, thus promoting a separation of roles.

\section{GRounding From TASK to Skill}

A CAD-level assembly specification must be refined to executable skills while respecting context-dependent constraints. Generic knowledge and rules to perform the refinement is stored in an ontology while the planning and inference module is responsible for selecting the most suitable skill and parameterize it such that the appropriate robot motion is produced. The following subsections describe in more details about the theory behind these two aspects.

\section{A. CAD-Based Ontology: From Task to Skill}

In order to ground an underspecified assembly task to executable skills, context-dependent information needs to be taken into account. One approach to this problem is to have the robot learn the necessary skills [19] [20]. In this work, we favor a

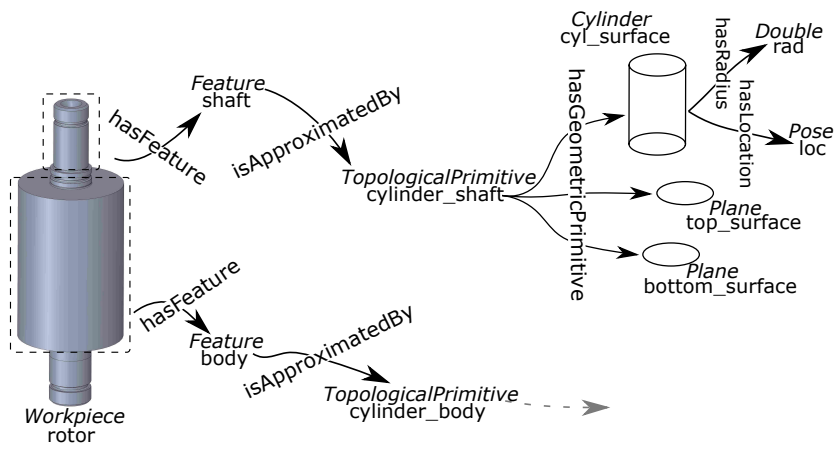

Fig. 2: The relational model describing a workpiece

knowledge-based approach where a general model of different entities involved in the task is stored in a knowledge database. An advantage of such an approach is the elimination of dataintensive and time-consuming learning processes thanks to the partial model of the real world encoded in the knowledge database. A widely used knowledge representation in the stateof-the-art literature [21] is the relational knowledge model, namely ontology.

Our ontology is based on the CAD semantics ontology proposed by Perzylo et al. in [1], extended with support for force-based assembly tasks. One of the main roles of the ontology is to store a relational model of workpieces. In this work, it is not necessary to provide a complete CAD model of the workpiece. Instead, it is sufficient to provide the CAD model of the workpiece's relevant assembly features. Such a paradigm allows more flexibility in modelling a workpiece because only features that are relevant to the task need to have the corresponding CAD model. This approach of workpiece modeling is illustrated in Fig. 2. A class is denoted with italic letters with the corresponding instance written below it. The arrows between classes denote the relations as they are defined in the ontology. The figure shows that a workpiece instance named rotor has two relevant features: shaft and body. Both features are approximated by BREP TopologicalPrimitive instances. A topological primitive is composed of geometric primitives, each with a link to the underlying CAD model. Classes such as Plane and Cylinder are subclasses of geometric primitive. The inference module can later query various numerical data from the geometric primitives such as the radius of a Cylinder instance. Note that although the workpiece rotor may have other features, they can be omitted if they are not relevant to the task specification.

Apart from modelling the workpiece, the ontology also models the relation between tasks and skills, as illustrated in Fig. 3. An assembly task is instantiated from the class Task. A task instance is composed of one or more $C A D$ Constraint instances. CADConstraint is a class that represents an assembly constraint between two geometric primitives. It can be further specialized into subclasses such as Concentric Constraint and CoincidentConstraint. On the other hand, a skill is instantiated from a Skill class and is composed of a number of SkillConstraint instances. These constraints are implemented in eTaSL and are imposed on robot controlled variables such as joint position or end-effector frame. For 


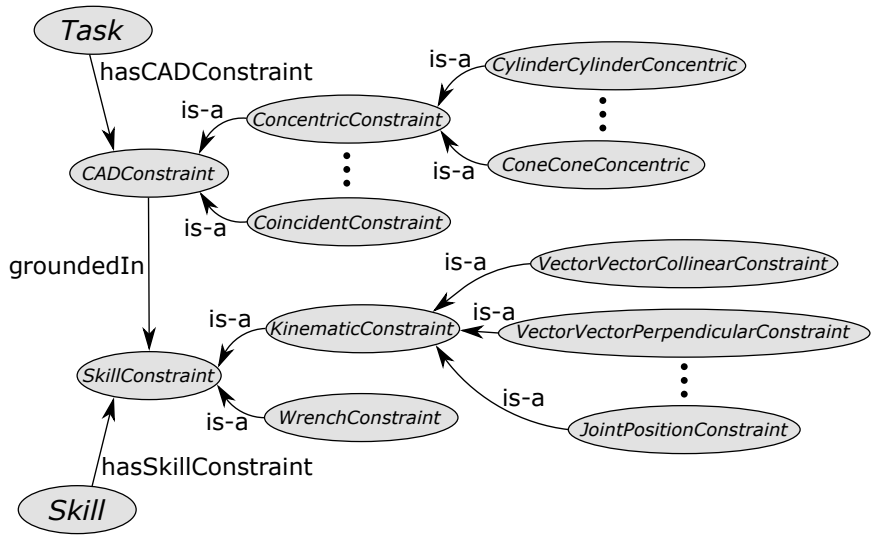

Fig. 3: A sub-ontology modeling the relation between task and skill

example the VectorVectorCollinearConstraint can be used to align the $z$-axis of the robot's end-effector frame to a target vector such as the axis of a cylindrical feature on a part.

A CADConstraint instance is related to SkillConstraint through the groundedIn property. This means that for a given assembly constraint defined in the workpiece perspective, there is at least one skill constraint defined in the robot perspective to execute it. By matching the CADConstraints comprising a task to the required SkillConstraints, the most suitable skill candidates can be inferred.

As indicated before, knowing which SkillConstraints to be used for achieving a given CADConstraint is crucial in the skill selection problem. For this purpose, the ontology covers an extensive knowledge of different mappings between these two classes. For tasks that only require position control, such as insertion with large enough clearance, KinematicConstraints should be used. Meanwhile, fine manipulation tasks require force-controlled skills i.e. skills composed of WrenchConstraints. We list some examples of these mappings in Table I. The complete mappings can be found in the ontology which is publicly available [22]. The table shows that, depending on the clearance between the two cylinders, the CylinderCylinderConcentric constraint can be realized either with a KinematicConstraint or a set of WrenchConstraints. For some subclasses of CADConstraint, however, it only makes sense to execute one class of skill constraint regardless of the context. The PlanePlaneCoincident, for example, can only be executed with wrench control since the robot's position control accuracy is not sufficient to ensure contact between two planes can be robustly made. Another example is the LinePointDistance which inherently does not involve any contact, hence only KinematicConstraint is suitable. In addition, the table also shows which WrenchConstraint directions are relevant. For instance, the CylinderCylinderConcentric requires force constraints along the $x, y$ and $z$ directions as well as torque constraints about the $x$ and $y$ directions.

\section{B. Planning and Inference Module}

The planning and inference module has three main purposes: plan the assembly sequence, select the skills based on the task, workpiece, and workcell model, and generate the appropriate skill parameters. Optimal planning of assembly involves optimal task sequencing, optimal trajectory planning, and selecting the optimal parameter values. These domains are interconnected, but they are assumed separable in the scope of this article.

As the tasks instantiated in the application layer describe the assembly from a workpiece perspective, they do not include information such as where the parts are in the workcell, or where the tools are. The planning and inference module includes sequential composition of motion skills, e.g. move and grip, move to assembly location, which allows to integrate such workcell information. Generating this skill sequence automatically is particularly challenging since it is tightly related to the assembly planning problem. Some of the difficulties are: ensuring that the skill sequence is geometrically valid, i.e., no interpenetration of parts, and respecting context-dependent constraints such as the gripper types or the reachability of the robot. Methods to localize parts in a workcell is not considered in this article, but the architecture aims for easy integration of localization systems.

Although assembly planning strategy is not the focus of this article, we outline below how an assembly planner may fit to the overall scheme.

1) Planning Module: Tasks and parts form a liaison graph. In liaison graphs, a node represents a part, and an edge represents an assembly situation [23]. A precedence graph specifies which edges in the liaison graph should be completed before others. This precedence graph represents all feasible assembly sequences. In [24], Homem De Mello and Sanderson show the relation between precedence graphs and other assembly representations such as And/Or graphs. The precedence graph is created in the application layer. Assembly of geometric features gives a defined assembly direction and virtual disassembly in the CAD software along these lines provides a suggested precedence graph. The precedence graph is passed from the application layer to the planner for generation of the assembly sequence. Moving to grip, changing tool, reorientation, and other workcell-related skills are added to the assembly sequence by the planner. Workcell information such as which parts and tools are available, or whether collaborative workspace skills are required, is an essential part of the planning module. This information must be stored in a robot cell database describing the different setups available. As the underlying control layer is robot-agnostic and the skills are transferable, we view this as a problem to be addressed in the planning module.

2) Inference Module: Given the assembly task instance, the inference module facilitates in grounding to the executable eTaSL skill. The role of the module is to infer the following:

- suitable skill candidates from the provided skill library

- the skill's application parameters

- the skill's process parameters

As robotized assembly is a complex problem with uncertainties and disturbances that are difficult to model, not all skills and their parameters can be inferred correctly. For example, non-geometric data such as the material properties, workpiece's weight and inertia are not taken into account in 
TABLE I: Examples of grounding maps between CAD constraints to the required skill constraints

\begin{tabular}{|c|c|c|}
\hline \multirow[b]{2}{*}{ CAD Constraint } & \multicolumn{2}{|c|}{ Skill Constraints } \\
\hline & KinematicConstraint & WrenchConstraint \\
\hline CylinderCylinderConcentric & 1 VectorVectorCollinearConstraint & 3 ForceConstraint and 2 TorqueConstraint \\
\hline PlanePlaneCoincident & $\mathrm{n} / \mathrm{a}$ & 1 ForceConstraint and 2 TorqueConstraint \\
\hline PlanePlaneParallel & 1 ForceConstraint and 2 TorqueConstraint & 1 VectorVectorParallelConstraint \\
\hline LinePointDistance & 1 PointVectorDistanceConstraint & $\mathrm{n} / \mathrm{a}$ \\
\hline
\end{tabular}

the task specification. Therefore, influence of these factors on the process parameters is not compensated. In this situation, knowledge from the user is still required. With this limitation in mind, we designed the inference module such that the operator's inputs can still be integrated. The interaction between the user and the inference module is illustrated by a flowchart shown in Fig. 4.

Once the task is instantiated and the workpieces to be assembled are defined, the inference module selects the most suitable skills. This is done by querying the ontology for skills whose eTaSL constraints match the task's CAD constraints according to the mapping described in Section IV-A. The implication of this strategy is that there may be more than one skill that can execute a given task. If such a situation occurs, the inference module informs the user to make the final skill selection.

The second purpose of the inference module is to generate application parameters that mainly depend on the geometric data contained in the CAD model. As an example case, consider the force-based assembly insertion shown in Fig. 5. Here, the relevant application parameters are the assembly direction $\mathrm{dir}$ and the insertion depth $h$. The assembly direction is inferred by checking the normal vectors of the bottom plane of the peg and the hole. Since the normal vectors are pointing outward of both the rotor and hole's solid, successful assembly can only be achieved when the two vectors have opposing directions. The assembly direction can be easily inferred by respecting this condition. The insertion depth is necessary for checking the skill status because the skill succeeds not only when the force along the $z$-axis reaches the target value, but also when the peg has been inserted with the correct depth. This way, a false positive error due to jamming can be detected.

The third role of the module is to infer the selected skill's process parameters. This is a difficult problem due to it being influenced by many different variables where good interaction

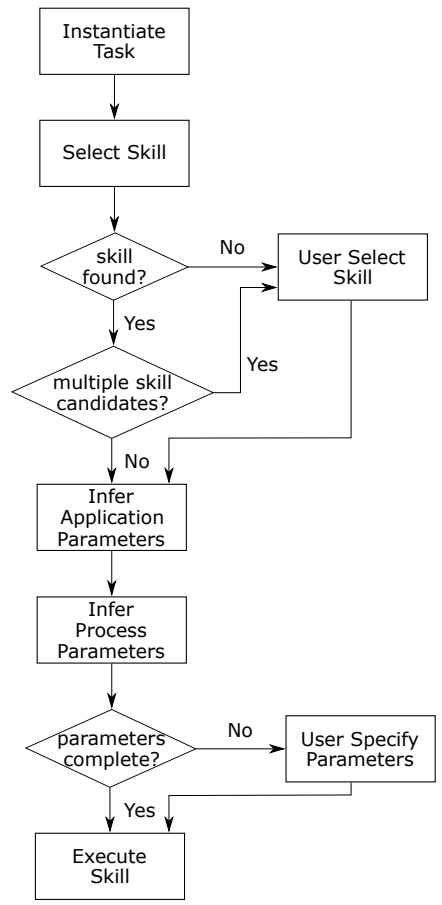

Fig. 4: Flowchart illustrating the steps in grounding from task to executable skill

models are usually not available. At its current state, our inference module can infer the process parameters required for insertion of a cylindrical object in a tight clearance situation. This task would be extremely difficult to perform with only position control, hence the necessity of a force-controlled skill. We generalize the task as a peg-in-hole problem.

A number of process parameters need to be tuned for an optimal peg-in-hole skill. From existing literature [25], it is known that the insertion behavior is determined by a number of factors such as the peg's dimension and the peg- 


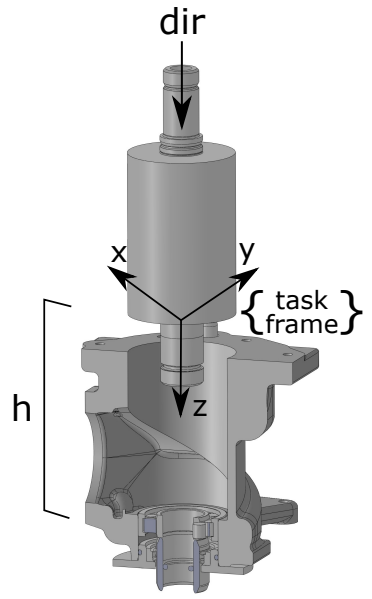

Fig. 5: Relevant application parameters in a peg-in-hole problem applied to a rotor assembly

hole clearance. Even for a peg-in-hole assembly with relatively simple geometry, accurate process modeling is difficult to achieve for narrow clearances. Due to hyperstatic contact situations, not all contact forces are always externally observable. We therefore use a data-driven approach to determine the process parameters. To infer the parameters in an uncertain or stochastic situation, a fuzzy inference approach is chosen [26]. Our fuzzy inference module uses the peg's length, peg's diameter and clearance to estimate the appropriate insertion force and dither amplitude.

\section{IMPLEMENTATION}

\section{A. Application Layer}

The application layer is implemented as a workbench in the open-source parametric CAD program FreeCAD 0.18 [27]. We implemented three task classes in the task library: Insert, Place, and Screw. Each task class is defined by a set of CAD constraints. Once the task is selected, the user annotates the CAD model with the relevant CAD constraints between features of two different parts. These CAD constraint instances together form the task instance. As task instances are defined between only two parts, we can automatically form a liaison diagram for the full assembly, see Fig.6. To transfer the information of the annotated tasks into the inference module, we implemented a tool for extracting the information as an OWL file. This automatically extracts the part-relative placement of the features, their geometric primitive class, and their geometric parameters such as normal of a plane, or the axis of a cylinder, as well as the constraints and tasks they are related with. The FreeCAD workbench also has tools for annotating relevant part-specific frames such as grasp frame as automatic generation of these is considered outside the scope of this article. These part-specific frames belong to a class called FeatureFrame and can be instantiated on a selected vertex, edge, or face. The parts are exported as STL meshes for visualization, and a JSON file describing the feature frames and the attributes of the geometric primitives relative to the mesh origin. The workbench is publicly available [28]. Since assembly sequence planning is not the focus of this paper, the assembly sequence was determined as the order in which tasks were annotated.

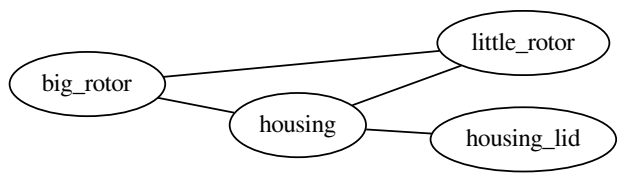

Fig. 6: Liaison diagram for the first assembly use case. Although the rotors are in contact with the lid, the chamfers allow us to use a place task defined between the housing and the lid.

\section{B. Process Layer}

1) Ontology: The ontology is implemented in the web ontology language (OWL) [29], a standard commonly used in robotics research [21] [30] [31]. We created the ontology using the Protégé software tool [32] which enables graph visualization and has a user-friendly interface for managing the ontology. One of the reasons for choosing OWL is because it allows for property restrictions which is important for mapping a CAD constraint to the corresponding skill constraint. We implemented the general knowledge described in Section IV-A in an OWL file.

2) Inference Module: For the skill selection and application parameter inference, a Prolog-based reasoner is implemented. Prolog is a language designed for logic programming and is particularly suitable for the first-order logic required in our application. Our inference module is written in SWIProlog [33] which features packages to load OWL files and parse them into Prolog's facts. We implemented a number of inference functions that reason using the stored facts.

As an example of how the inference module resolves the skill selection problem, we queried for the suitable skill candidates for the rotor assembly task illustrated in Fig. 5. This task can be specified with a cylindrical insertion task given in Listing 1 . The function call and the returned skills is provided in Listing 2 with Prolog-like syntax. The function get_suitable_skill takes the task instance RotorAssemblyTask as an argument and returns the Skills variable. The function infers that there are three suitable skill candidates to execute the task. Apart from the obvious CylindricalInsert skill, the ApplyWrench skill is suitable because the skill controls forces and torques on all six axes of a given task frame. By applying the correct target values on each force and torque axis, similar behavior to Cylindricalinsert is obtained. Furthermore, the NonCylindricalinsert is also a valid candidate since the skill has the same constraints as Cylindricalinsert with additional torque control along the $z$-axis. Due to the symmetry of a cylinder, this presence of this extra constraint does not affect the end-goal of the task. 
Listing 2: Inferring skill candidates for rotor assembly task get_suitable_skill('RotorAssemblyTask', Skills).

Skills = 'ApplyWrench';

Skills = 'CylindricalInsert';

Skills $=$ 'NonCylindricalInsert'

In order to infer the appropriate process parameter values for the cylindrical peg-in-hole task, a Mamdani-type fuzzy inference is implemented in MATLAB R2017a's fuzzy logic designer toolbox. We defined in total 21 rules describing the mapping from the peg's dimension and the clearance to the appropriate insertion force and dither amplitude. The fuzzy rules are derived from rough modeling of the contact situation and the empirical trend observed after executing the cylinder insertion skill. Each fuzzy rule is approximated with Gaussian membership functions that categorize a range of parameter values into fuzzy sets. For example, the peg's diameter can be categorized into "very small", "small", "medium", "large" and "very large". The fuzzy sets for each geometric and process parameters are shown in Fig. 7 while five sample rules are provided in Listing 3.

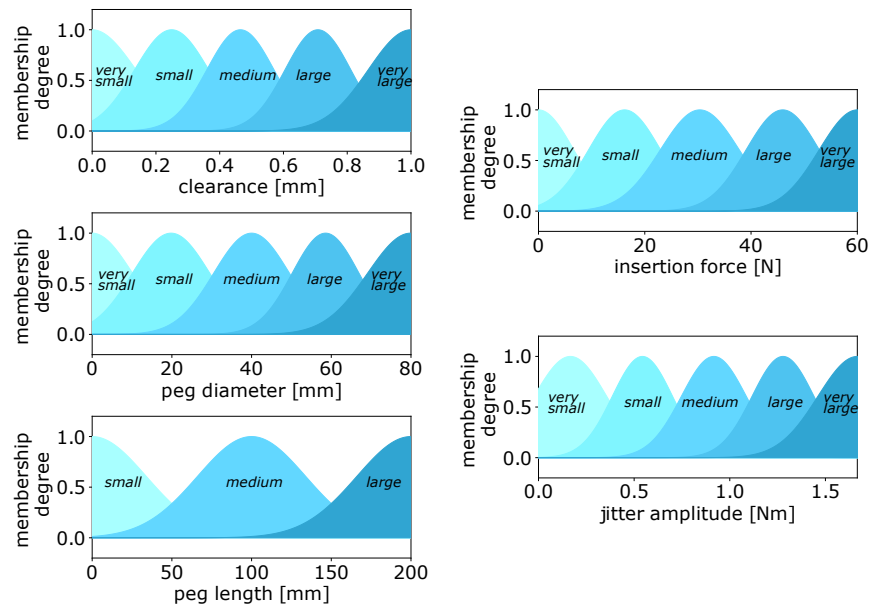

Fig. 7: The fuzzy sets used in the inference module. The left column shows the geometric parameters while the right one shows the process parameters.

Listing 3: Selection of the fuzzy rules used in the inference module

if diameter is "small" then insertion force is "large"

if clearance is "medium" then insertion force is "medium"

if length is "long" then insertion force is "small"

if clearance is "very small" then dither amplitude is "very large"

if diameter is "small" and length is "long" then dither amplitude is "medium"

In this data-driven approach, we first collected the training data by running a total of 40 peg-in-hole trials with different process parameter values. For each run, the insertion force and dither amplitude were carefully selected to ensure successful execution, while minimizing contact force. Based on these experiments, the number of membership functions and their shapes were then tuned. Finally, the fuzzy inference module was validated with a new set of peg-in-hole tasks.

3) Implemented Skills: Our skill library consists of around 20 skills, and the skill semantics are stored in the ontology.

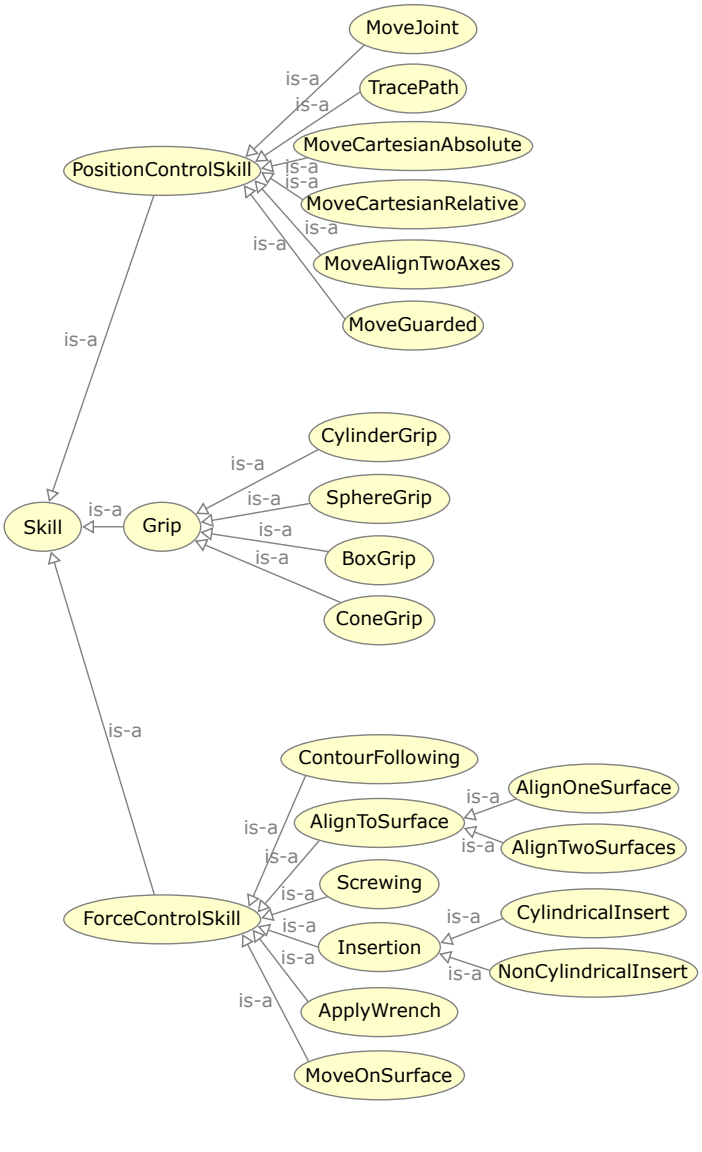

Fig. 8: The skill taxonomy as defined in the OWL ontology.

The ontology organizes the skills according to different taxonomies e.g. whether a skill belongs to PositionControlSkill or ForceControlSkill class. Fig. 8 shows a graph visualization of the skill taxonomies generated by the Protégé tool.

For executing a given assembly task, not only skills to perform the assembly during interaction are needed, but also skills to perform the free-space motion of the pre- and postassembly action. This includes skills to grip the workpiece and skills to retract the robot once the assembly is finished. These skills are workcell dependent, but common for all workpieces. As assembly planning is not the focus of this article, the inference module presented in this article only deals with selecting and generating the parameters for the actual assembly skill, i.e. the robot motion that achieves the task-specific CAD constraints. However, as pointed out in Section IV-B, the intended behavior of the proposed system is to easily integrate existing planning modules such that the pre- and post-assembly skills are composed automatically while taking into account the workcell context. For this reason, in our current implementation, the pre- and postassembly skills are preselected manually and configured with the parameters obtained from the CAD model and querying the relative transformations between relevant feature frames. For example, to move the gripper to the workpiece location, MoveCartesianAbsolute is preselected, while the target pose of the gripper is obtained from the part's feature frame.

To coordinate the skills from gripping, inserting to finally 


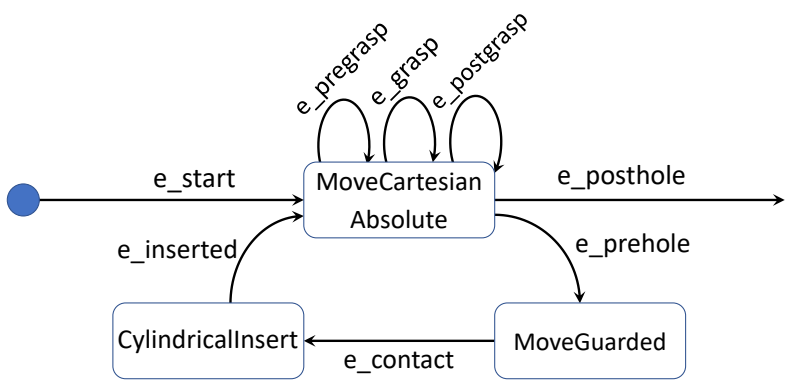

Fig. 9: Example of the finite state machine for gripping and inserting a workpiece. The transition between two states is triggered by incoming event "e_event_name"

retracting, we used the restricted finite state machine (rFSM) library [34]. Whenever a skill finishes or fails, its monitor will trigger an event message to the rFSM coordinator and the transition to the subsequent skill will be made.

A common approach in robotics is to have states in the FSM denote the motion goals e.g. move_to_grip_pose. This puts the skill complexity in the states. An approach more often found in computer games is to have states denote operational modes such as walk and run, and then redefine the parameters of these modes during runtime. This reduces the states used and puts the complexity in the transitions. We have used the operational modes approach as it is easy to reuse an FSM with new skill parameters, and as transition events denote the momentary situation that the robot is in. Therefore, each rFSM's state corresponds to a skill to be executed, and the state transition reconfigures the skill with the updated application and process parameters. An example of the rFSM to grip and assemble a workpiece is shown in Fig. 9. When $e_{-}$start occurs, we set the target pose of MoveCartesianAbsolute to an offset distance above the workpiece's pick frame. Success in reaching this target pose is associated with the e_pregrip event. When e_pregrip occurs the target pose of MoveCartesianAbsolute is updated to the part's pick frame and $e_{-}$grip event is emitted upon success. When e_grip occurs the robot has reached the workpiece's pick frame, engaged the gripper and set the next target pose to a $z$-axis offset from the pick frame, and success gives the $e_{-}$postgrip event. The procedure is repeated for the $z$-axis pre-hole locations. When $e$ prehole occurs we transition into MoveGuarded which is a Cartesian motion towards the hole with monitors that trigger when the endeffector force exceeds a threshold. Once contact between the workpiece and hole is made, signaled by the $e_{-}$contact event, the CylindricalInsert skill is executed. This procedure of reconfiguring the skill parameters is adhered to for all of the transitions.

\section{Control Layer}

In this article, we present a more extensive use cases by testing our system on two different robot setups with different communication interfaces. The first setup consists of a seven DOF KUKA LBR iiwa robot equipped with a pneumatic SCHUNK RH9010 parallel gripper. The LBR iiwa is controlled over Fast Robot Interface (FRI) [35] with a control loop frequency of $200 \mathrm{~Hz}$. This robot provides joint torques measurements and estimates of the external end effector wrench (force/moment), which can be used in the execution of force-based skills.

The second setup consists of a KUKA KR16-2, a larger industrial robot with lower control frequency of $83 \mathrm{~Hz}$ controlled using the Robot Sensor Interface (RSI). Since the KR16 does not have any torque sensor, we attached a Schunk 6D Force/Torque sensor on the end-effector. Both robots are controlled with eTaSL/eTC [2] which is deployed as an Orocos [36] component. eTaSL is used for constraint specification in Lua language while eTC is the controller implementation that ensures exponential convergence of the constraints in an instantaneously optimal way. Additionally, a feature_frame_publisher node [37] was created in ROS [38] for publishing all relevant frames with TF2.

\section{Test CAses}

This section describes two test cases that were performed to investigate the prototype architecture. The goal of the test cases is to validate that the implemented prototype works well in specifying the CAD-level assembly task as well as generating the executable skills.

Each test case is carried out according to the following steps. For every part to be assembled, the programmer annotates the relevant features of the part and selects the desired task class though the FreeCAD interface. Once the task instance is created, the most suitable skill class is deduced by the Prologbased reasoner and the appropriate process parameters are generated by the inference module. The geometry of the parts such as the dimension and the clearance are different so as to verify the effectiveness of the process parameters inference. Once an executable skill is instantiated, an eTaSL controller is set to execute it.

\section{A. Case 1: Assembly of Air Compressor}

We consider an assembly of a rotary-screw compressor (see Fig.10). The compressor is composed of over 30 parts. To limit the scope of this demonstration, we focus on the assembly of the rotors and the lid. The housing is attached to a fixture and all insertions are in the same direction. The compressor has a small and large meshing helical screw rotors. The upper shaft of the rotors go through the lid through two chamfered holes. All parts to be assembled are placed in known poses.

The assembly of the two rotors is done with the Insert task while the lid is performed with the Place task. The Insert task is specified with a set of CAD constraints reported in Listing 1. The Place task is specified with two CylinderCylinderConcentric constraints so as to align the two rotors' shafts with the lid's holes and an AxisVelocityConstraint to bring the lid down to the housing with a constant velocity.

Since the two rotors and holes have tight clearance, the task should be executed with force-controlled skills. The inference module correctly deduces the suitable skill candidate to be Cylindricallnsert skill, infers the assembly direction and insertion length, and generates the process parameters presented in Table II. As for the lid, there is sufficient clearance 
for the robot to accurately perform the assembly without using force control. Therefore, the task is solved by executing a position control skill MoveAlignTwoAxes (see Fig. 8) with force threshold monitor to detect contact.

The experiment shows that the robot successfully assembles the parts using the skill and parameters inferred by the inference module, see Fig. 11 for a snapshot of the assembly sequence. The recorded insertion forces and moments during a portion of the assembly sequence are shown in Fig 12. The graph gives a recording of the transition from MoveGuarded to CylindricalInsert.

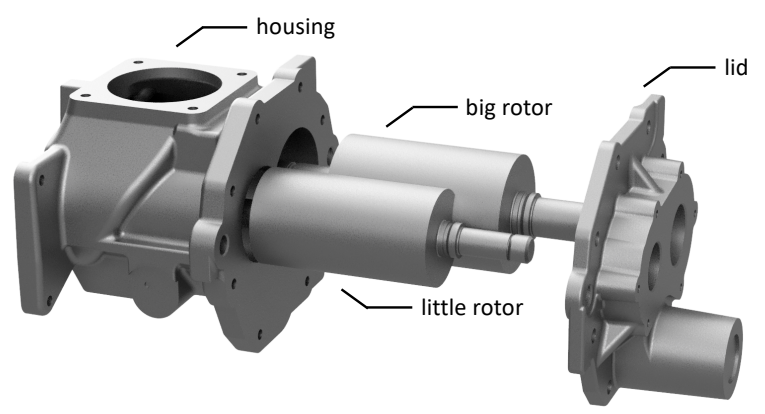

Fig. 10: CAD model of the air compressor. Note that the helical screw profile is not modeled.

TABLE II: Geometric and Inferred Process Parameters for the Compressor Rotor Assemblies

\begin{tabular}{cccc}
\hline \multirow{2}{*}{ Parameter Category } & Parameter Name & small_rotor & big_rotor \\
\hline \multirow{3}{*}{ Geometric } & Length $(\mathrm{mm})$ & 192 & 192 \\
& Diameter $(\mathrm{mm})$ & 61 & 75.5 \\
& Clearance $(\mathrm{mm})$ & 0.1 & 0.08 \\
\multirow{2}{*}{ Process } & Insertion Force $(\mathrm{N})$ & -31.1 & -33.5 \\
& Dither Amplitude $(\mathrm{Nm})$ & 1.13 & 1.33 \\
\hline
\end{tabular}
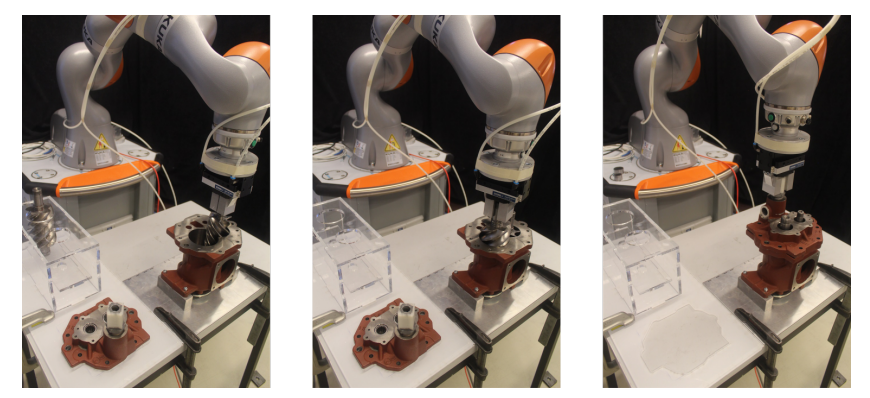

Fig. 11: The robot performing the assembly sequence of the compressor parts. From left to right: assembly of the small rotor, the big rotor, and the housing lid.

\section{B. Case 2: Assembly of Electrical Motor}

In this example we consider the assembly of an electrical motor (see Fig. 13) by peg-in-hole insertion of the rotor. This is similar to the previous example but showcases the robotagnostic nature of the control-layer as it is controlling a KUKA
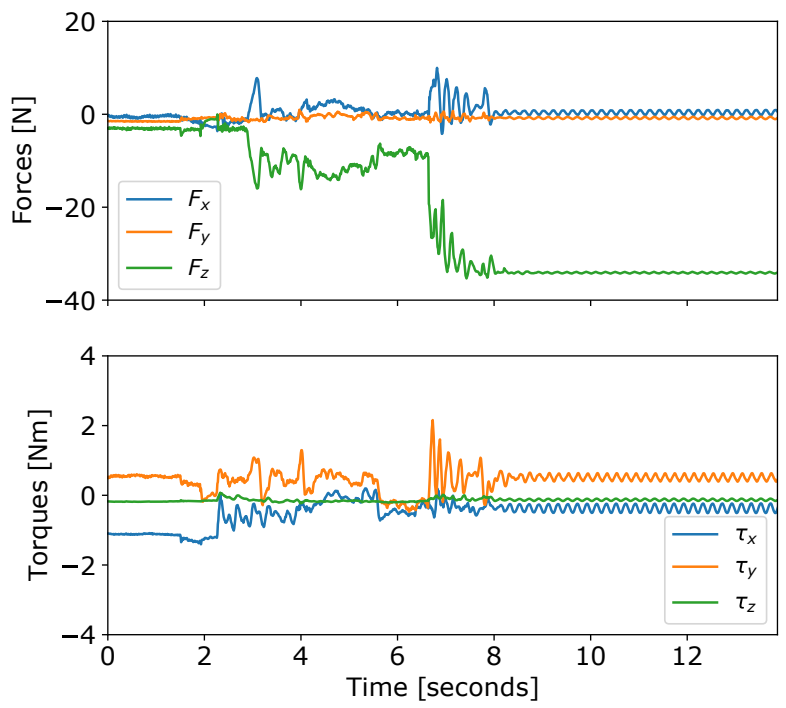

Fig. 12: The measured forces and torques during insertion of the big rotor. Note that from $8 \mathrm{~s}$, the rotor is in contact with the bottom. This was added to verify that the desired dithering is present.

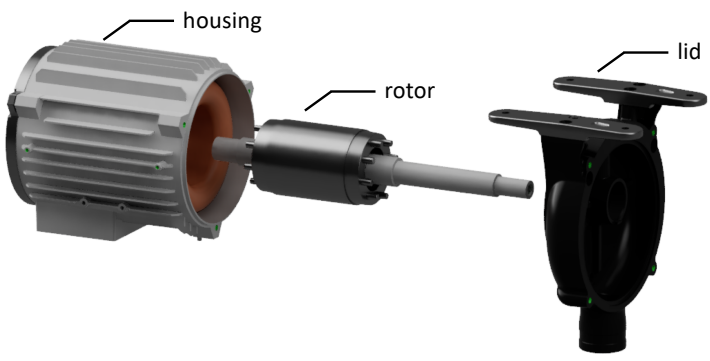

Fig. 13: CAD model of the electrical motor.

KR16-2, illustrated in Fig. 14, with a high mechanical stiffness and a slower communication interface. The employed skills are the same as for the rotary screw compressor example, with process parameters generated by the inference module as shown in Table III.

In Fig. 15 the measured forces at the end-effector are given for the transition from MoveGuarded to CylindricalInsert, and a moment while it remains in CylindricalInsert after the skill is achieved to show the behavior once contact occurs. The gain of the force constraint in assembly direction has been lowered to reduce the overshoot and movement speed of the robot. Monitors on insertion distance, forces, and torques have been removed to observe the continuous behavior of CylindricalInsert under the higher mechanical stiffness and slower controller rate.

\section{DISCUSSION}

The force controller in eTaSL has a damping behavior [2]. This resulted in a velocity proportional to the difference between the measured and desired force. For the first use case, Fig. 12 shows that when the robot approached the rotor's hole 


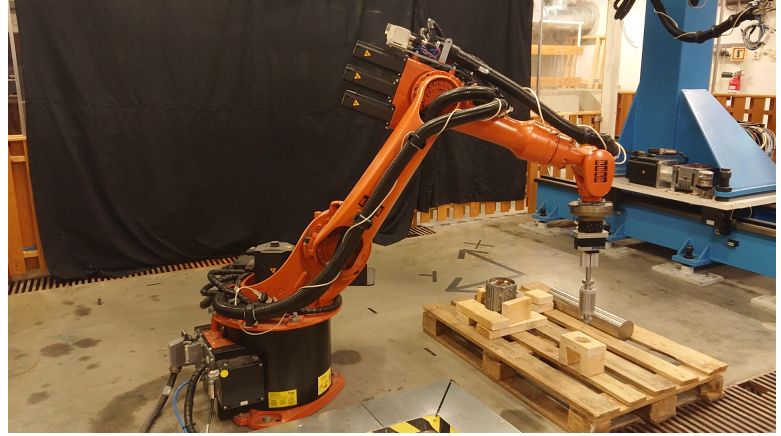

Fig. 14: KR16-2 performing the assembly sequence of the electrical motor.

TABLE III: Geometric and Inferred Process Parameters for the Motor Assemblies

\begin{tabular}{ccc}
\hline \multirow{2}{*}{ Parameter Category } & Parameter Name & $\begin{array}{c}\text { Part Name } \\
\text { rotor }\end{array}$ \\
\hline \multirow{2}{*}{ Geometric } & Length $(\mathrm{mm})$ & 74 \\
& Diameter $(\mathrm{mm})$ & 64 \\
& Clearance $(\mathrm{mm})$ & 0.5 \\
Process & Insertion Force (N) & -37.4 \\
& Dither Amplitude (Nm) & 0.74 \\
\hline
\end{tabular}

between $0 \mathrm{~s}$ and $2.8 \mathrm{~s}$ the forces were approximately zero. When a force magnitude of $-10 \mathrm{~N}$ in $F_{z}$ was experienced by MoveGuarded, a transition into CylindricalInsert occurred. The insertion process lasted from $2.8 \mathrm{~s}$ to $6.7 \mathrm{~s}$ and the robot was moving down while trying to achieve the target insertion force. The effects of friction (approximately -10 $\mathrm{N}$ in $z$-direction) are visible during the insertion. From 6.7 $\mathrm{s}$ to $8 \mathrm{~s}$, the rotor touched the bottom of the hole, the velocity dropped to zero, and the set-point for the insertion force reached its desired value. The measured force along the $z$-axis was consistent with the inferred process parameters listed in Table II. Furthermore, the oscillations due to the applied dither-force remained bounded. This shows that the skill executed the assembly task as desired.

The monitors in eTaSL trigger switching of skills for safer execution. The KR16-2 has a motion buffer that introduces a tracking delay [39]. When a force controlled skill such as Cylindricallnsert is run, the tracking delay can cause an overshoot from the desired force as seen in Fig. 15, but the system converges to the desired force in the end. In the test presented in the figure, the gain of the compliance constraint has been lowered so as to lower the movement speed of the robot, and monitors to indicate successful execution of the skill have been removed. When the monitors are present, the skill will switch at first available sensor signal indicating failure or success condition. This ensures a safer execution of forcecontrolled skills.

In this paper we have emphasized the geometric information available in the CAD data, but there is another aspect that is of relevance, the material properties of the parts involved. For example, insertion of a teflon peg in a steel hole has different process parameters to inserting a steel peg in a soft-plastic hole. Material properties are available in STEP AP214 file

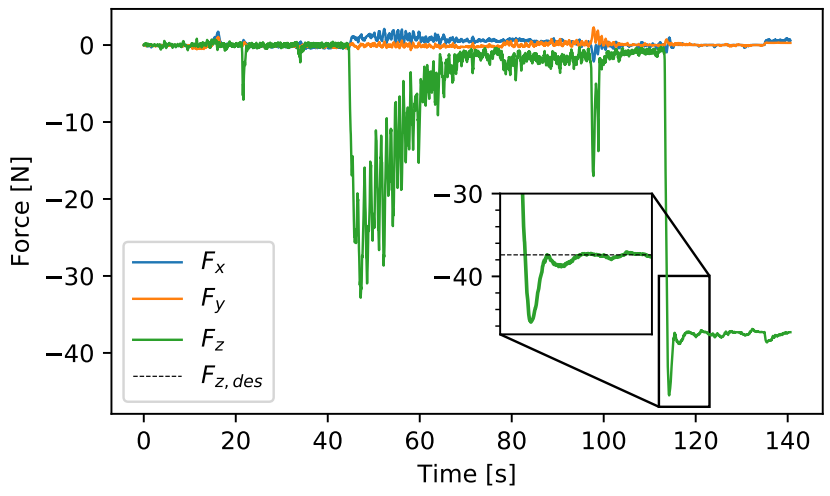

Fig. 15: The measured forces when inserting the rotor in the motor housing. Note that this example moves slower as it uses a lower gain to show CylindricalInsert behavior. The inset axes show the overshoot but eventual convergence to the desired insertion force.

format, and many CAD programs support it. For multi-material assembly scenarios, the inference module should be trained on the material properties as well as the geometric properties. The newer STEP AP242 format includes methods for annotating physical surface characteristics of features as well which can further improve the inference engine [40].

Although this paper does not quantify the benefit of the proposed architecture, such as how much the programming time is reduced, the preliminary evaluation shows promising benefits for potential users. The current industry practice for programming assembly tasks is a robot-centric approach. Not only does this make the program often not reusable, the level of robotics expertise needed is considerably high. Programmers have to write low-level code such as implementing the impedance controller for force-sensitive tasks. The architecture proposed in this paper alleviates this problem by facilitating task-centric specifications that liberate users from low-level coding. Therefore, users such as application deployers who are experts in the assembly process can specify the task in the abstraction level that they are familiar with. Furthermore, the use cases across different robot models with the same skill library suggests better reusability than the current industry practice.

\section{CONCLUSION}

In this paper, we extend the system architecture proposed in our prior research [3] by presenting an improved methodology to automatically select a suitable skill and its corresponding parameters to execute a given assembly task. This improvement is made possible by modeling the semantics of relevant classes involved in the assembly context such as geometric primitives, tasks and skills, as well as building an ontology to model their relations. The architecture is split into three layers. The application layer allows the user to annotate tasks according to a task specification using CAD constraints. The process layer infers the appropriate skill and generates both application and process parameters using a Prolog-based reasoner and 
fuzzy-inference. Finally, the control layer executes the task in the eTaSL framework with an rFSM-based state machine to coordinate the skill transitions. The system architecture is designed such that the task is executed in a robot-agnostic manner.

The proposed system is validated on challenging assembly tasks that require force-control. The inference module is shown to correctly select the suitable force-based skills to successfully execute tight-tolerance tasks. Apart from the geometryrelated application parameters, the selected skills require process parameters such as insertion force and dither amplitude. These empirical parameters are not readily available in the CAD model. Therefore, a fuzzy inference method is employed to estimate these parameters based on a set of key geometric parameters from the CAD model.

The experimental validation is conducted on two different assembly problems performed by two different robot setups: assembly of an air compressor and an electric motor. In both cases, the rotor assemblies can be generalized as a peg-inhole task, for which the conventional solutions have been studied well [41]. The key geometric parameters used in the fuzzy inference were the peg's clearance, peg's diameter, and insertion length, all of which were extracted from the CAD model. For more complex assembly tasks, such as snap-fit, the key geometric parameters are yet to be defined.

A limitation of the fuzzy inference method is that it fails to extrapolate outside the predefined fuzzy set range, hence the dataset should vary enough to cover the given workpiece geometry. Furthermore, finding the relevant geometric parameters for a given assembly task can be challenging. However, with a sufficiently large dataset and more advanced regression methods, such as automatic relevance detection [42], we argue that it is possible to automatically deduce which of the parameters are really relevant for the process parameters. Such an approach will be beneficial for including material properties when inferring process parameters. The fuzzy set database can grow over time to accommodate a larger variety of assembly cases and this database can be shared with similar robot setups.

The software implementation serves as a proof-of-concept that validates the intended goal of the proposed system architecture. The assembly of the rotors and housing lid was successfully executed by the system, and the inference module provided reasonable parameters. The application layer implementation is used for instantiating assembly tasks through annotation of the CAD model's geometric features and the publisher node gives the relevant feature frames transformation in ROS.

\section{ACKNOWLEDGMENT}

The work reported in this paper was supported by Flanders Make ICON FINROP (Fast and Intuitive Robot Programming) and SBO PROUD (Programming by User Demonstration) in Belgium and the centre for research based innovation SFI Manufacturing in Norway. The work is partially funded by the Research Council of Norway under contract number 237900. The authors would like to thank Atlas Copco Airpower NV and Mjøs Metallvarefabrikk for providing the assembly use cases.

\section{REFERENCES}

[1] A. Perzylo, N. Somani, M. Rickert, and A. Knoll, "An ontology for cad data and geometric constraints as a link between product models and semantic robot task descriptions," in 2015 IEEE/RSJ International Conference on Intelligent Robots and Systems (IROS), Sep. 2015, pp. 4197-4203.

[2] E. Aertbelien and J. De Schutter, "eTaSL/eTC: A constraint-based task specification language and robot controller using expression graphs," in 2014 IEEE/RSJ International Conference on Intelligent Robots and Systems. IEEE, sep 2014, pp. 1540-1546.

[3] M. H. Arbo, Y. Pane, E. Aertbeliën, and W. Decré, "A System Architecture for Constraint-Based Robotic Assembly with CAD Information," in IEEE 14th International Conference on Automation Science and Engineering (CASE'18), Munich, aug 2018, pp. 690-696.

[4] A. Ambler and R. Popplestone, "Inferring the positions of bodies from specified spatial relationships," Artificial Intelligence, vol. 6, no. 2, pp. 157-174, jun 1975.

[5] L. I. Lieberman and M. A. Wesley, "AUTOPASS: An Automatic Programming System for Computer Controlled Mechanical Assembly," IBM Journal of Research and Development, vol. 21, no. 4, pp. 321-333, jul 1977.

[6] S. Kaufman, R. Wilson, R. Jones, T. Calton, and A. Ames, "The Archimedes 2 mechanical assembly planning system," in Proceedings of IEEE International Conference on Robotics and Automation, vol. 4. IEEE, 1996, pp. 3361-3368.

[7] H. Mosemann and F. M. Wahl, "Automatic Decomposition of Planned Assembly Sequences Into Skill Primitives," IEEE Transactions on Robotics and Automation, vol. 17, no. 5, pp. 709-718, 2001.

[8] U. Thomas and F. M. Wahl, "Assembly Planning and Task Planning - Two Prerequisites for Automated Robot Programming," in Springer Tracts in Advanced Robotics, 2010, vol. 67, pp. 333-354.

[9] F. Dietrich, J. Maaß, A. Raatz, and J. Hesselbach, "RCA562: Control Architecture for Parallel Kinematic Robots," in Springer Tracts in Advanced Robotics, 2010, vol. 67, pp. 315-331.

[10] U. Thomas, F. Wahl, J. Maass, and J. Hesselbach, "Towards a new concept of robot programming in high speed assembly applications," in IEEE/RSJ International Conference on Intelligent Robots and Systems (IROS'05), 2005, pp. 3827-3833.

[11] A. Perzylo, N. Somani, S. Profanter, I. Kessler, M. Rickert, and A. Knoll, "Intuitive instruction of industrial robots: Semantic process descriptions for small lot production," in 2016 IEEE/RSJ International Conference on Intelligent Robots and Systems (IROS), vol. 2016-Novem. IEEE, oct 2016, pp. 2293-2300.

[12] N. Somani, A. Gaschler, M. Rickert, A. Perzylo, and A. Knoll, "Constraint-based task programming with cad semantics: From intuitive specification to real-time control," in 2015 IEEE/RSJ International Conference on Intelligent Robots and Systems (IROS), Sep. 2015, pp. 2854-2859

[13] A. Perzylo, M. Rickert, B. Kahl, N. Somani, C. Lehmann, A. Kuss, S. Profanter, A. B. Beck, M. Haage, M. Rath Hansen, M. T. Nibe, M. A. Roa, O. Sornmo, S. Gestegard Robertz, U. Thomas, G. Veiga, E. A. Topp, I. Kessler, and M. Danzer, "Smerobotics: Smart robots for flexible manufacturing," IEEE Robotics Automation Magazine, vol. 26, no. 1, pp. 78-90, March 2019.

[14] A. Kuss, J. R. Diaz P., R. Hollmann, T. Dietz, and M. Hägele, "Manufacturing knowledge for industrial robot systems: Review and synthesis of model architecture," in 2016 IEEE International Conference on Automation Science and Engineering (CASE), Aug 2016, pp. 348354.

[15] U. Thomas, G. Hirzinger, B. Rumpe, C. Schulze, and A. Wortmann, "A new skill based robot programming language using uml/p statecharts," in 2013 IEEE International Conference on Robotics and Automation, May 2013, pp. 461-466.

[16] M. T. Mason, "Compliance and Force Control for Computer Controlled Manipulators," IEEE Transactions on Systems, Man, and Cybernetics, vol. 11, no. 6, pp. 418-432, 1981.

[17] J. De Schutter, T. De Laet, J. Rutgeerts, W. Decré, R. Smits, E. Aertbeliën, K. Claes, and H. Bruyninckx, "Constraint-based Task Specification and Estimation for Sensor-Based Robot Systems in the Presence of Geometric Uncertainty," The International Journal of Robotics Research, vol. 26, no. 5, pp. 433-455, may 2007. 
[18] R. Smits, T. De Laet, K. Claes, H. Bruyninckx, and J. De Schutter, "iTASC: a tool for multi-sensor integration in robot manipulation," in 2008 IEEE International Conference on Multisensor Fusion and Integration for Intelligent Systems. IEEE, aug 2008, pp. 426-433.

[19] S. Levine, N. Wagener, and P. Abbeel, "Learning contact-rich manipulation skills with guided policy search," in 2015 IEEE International Conference on Robotics and Automation (ICRA), May 2015, pp. 156163.

[20] M. Kalakrishnan, L. Righetti, P. Pastor, and S. Schaal, "Learning force control policies for compliant manipulation," in 2011 IEEE/RSJ International Conference on Intelligent Robots and Systems, Sep. 2011, pp. 4639-4644.

[21] M. Tenorth and M. Beetz, "Representations for robot knowledge in the KnowRob framework," Artificial Intelligence, vol. 247, pp. 151-169, 2017.

[22] Y. Pane and M. H. Arbo, "CAD Based Assembly Ontology," 2019. [Online]. Available: https://github.com/yudhapane/cad_based_ assembly_ontology

[23] T. De Fazio and D. Whitney, "Simplified generation of all mechanical assembly sequences," IEEE Journal on Robotics and Automation, vol. 3 , no. 6, pp. 640-658, dec 1987.

[24] L. Homem de Mello and A. Sanderson, "Representations of mechanical assembly sequences," IEEE Transactions on Robotics and Automation, vol. 7, no. 2, pp. 211-227, apr 1991.

[25] N. Pitchandi, S. P. Subramanian, and M. Irulappan, "Insertion force analysis of compliantly supported peg-in-hole assembly," Assembly Automation, vol. 37, no. 3, pp. 285-295, 2017.

[26] S. Guillaume, "Designing fuzzy inference systems from data: An interpretability-oriented review," IEEE Transactions on Fuzzy Systems, vol. 9, no. 3, pp. 426-443, jun 2001.

[27] J. Riegel and Y. van Havre, "FreeCAD: Parametric 3D modeler." [Online]. Available: https://www.freecadweb.org

[28] M. H. Arbo and Y. Pane, "ARBench," 2017. [Online]. Available: https://github.com/mahaarbo/ARBench

[29] G. Antoniou and F. Van Harmelen, "Web ontology language: Owl," in Handbook on Ontologies in Information Systems. Springer, 2003, pp. 67-92.

[30] M. Stenmark and J. Malec, "Knowledge-based instruction of manipulation tasks for industrial robotics," Robotics and Computer-Integrated Manufacturing, 2014.

[31] M. Waibel, M. Beetz, J. Civera, R. D’Andrea, J. Elfring, D. GálvezLópez, K. Häussermann, R. Janssen, J. M. M. Montiel, A. Perzylo, B. Schießle, M. Tenorth, O. Zweigle, and R. V. De Molengraft, "Roboearth," IEEE Robotics Automation Magazine, vol. 18, no. 2, pp. 69-82, June 2011

[32] M. A. Musen, "The Protégé Project: A Look Back and a Look Forward," AI Matters, pp. 4-12, 2015.

[33] J. Wielemaker, T. Schrijvers, M. Triska, and T. Lager, "SWI-Prolog," Theory and Practice of Logic Programming, vol. 12, no. 1-2, pp. 6796, 2012.

[34] M. Klotzbücher and H. Bruyninckx, "Coordinating robotic tasks and systems with rfsm statecharts," JOSER: Journal of Software Engineering for Robotics, vol. 3, pp. 28-56, 2010.

[35] G. Schreiber, A. Stemmer, and R. Bischoff, "The fast research interface for the kuka lightweight robot," in IEEE ICRA 2010 Workshop on Innovative Robot Control Architectures for Demanding (Research) Applications, 2010

[36] P. Soetens, "A Software Framework for Real-Time and Distributed Robot and Machine Control," Ph.D. dissertation, Katholieke Universiteit Leuven, 2006

[37] M. H. Arbo and Y. Pane, "ARBench part publisher," 2017. [Online]. Available: https://github.com/mahaarbo/arbench_part_publisher

[38] M. Morgan Quigley, B. Gerkey, K. Conley, J. Faust, T. Foote, J. Leibs, E. Berger, R. Wheeler, and A. Ng, "ROS: an open-source Robot Operating System," in Proc. of the IEEE Intl. Conf. on Robotics and Automation (ICRA) Workshop on Open Source Robotics, may 2009.

[39] M. Lind, J. Schrimpf, and T. Ulleberg, "Open Real-Time Robot Controller Framework," in 3rd CIRP Conference on Assembly Technology and Systems (CATS'10), Trondheim, 2010, pp. 13-18.

[40] S. A. Organization, "AP242 - Why the convergence." [Online]. Available: http://www.ap242.org/why-ap242

[41] T. Lozano-Perez, M. T. Mason, and R. H. Taylor, "Automatic synthesis of fine-motion strategies for robots," The International Journal of Robotics Research, vol. 3, no. 1, pp. 3-24, 1984.

[42] C. M. Bishop, Pattern Recognition and Machine Learning. New York: Springer, 2006.

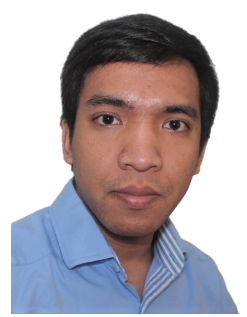

Yudha Pane received the B.Sc. degree in electrical engineering from Bandung Institute of Technology, Indonesia in 2013, and the M.Sc. degree in systems and control from Delft University of Technology, The Netherlands in 2015. Since 2016, he is a Ph.D. student at KU Leuven Robotics Research Group. $\mathrm{He}$ received the best student paper award from the 14th IEEE Conference on Automation Science and Engineering (CASE) in 2018. His research interests include skill-based robot programming and control of mechatronics systems.

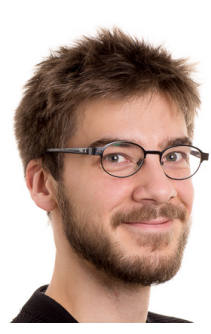

Mathias Hauan Arbo received the M.Sc. degree and the Ph.D. degree in Engineering Cybernetics from the Norwegian University of Science and Technology (NTNU), Trondheim, Norway in 2015 and 2019 respectively. He is currently employed as a postdoc at the NTNU through SFI Manufacturing. He received the best student paper award from the 14th IEEE Conference on Automation Science and Engineering (CASE) in 2018. His research interests include robotic assembly, optimization-based control, force control, and motion control.

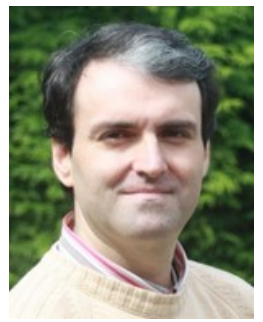

Erwin Aertbeliën received the M.Sc. Degree and the Ph.D. degree in mechanical engineering from KU Leuven, Belgium in 1995 and 2009, respectively. He was appointed research expert in 2011 and since 2016 , he is a senior research expert with expertise in automation and robotics. He has been involved in various EU projects such as Echord (Containerbot), ROSETTA, and Factory-in-a-day. His research interests are in force controlled robotics, human-intent estimation and control for lower-limb exoskeletons, and programming by demonstration for manipulation and assembly. He is also the developer of the constraint-based robotic task specification framework eTaSL.

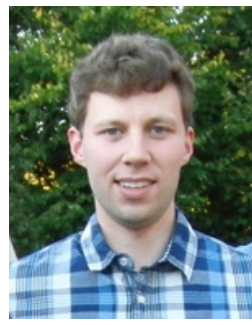

Wilm Decré received the M.Sc. degree in Mechanical Engineering and the Ph.D. degree in Engineering from KU Leuven, Belgium in 2006 and 2011, respectively. In 2006 he received the best mechanical engineering thesis prize from LMS International; in 2007 he was first laureate of the IEEE Region 8 Best Student Paper Contest. Currently he is focusing on the industry-oriented research activities of the Robotics and MECO (Motion, Estimation, Control and Optimisation) research teams as a research manager. He is and/or was involved in European and Belgian projects on sensor-based and optimisation-based robot control, human-intent estimation and programming by human demonstration. 